\title{
DOUBLING AND TRIPLING CONSTRUCTIONS FOR DEFINING SETS IN STEINER TRIPLE SYSTEMS
}

\author{
DIANE DONOVAN, ABDOLLAH KHODKAR AND ANNE PENFOLD STREET \\ CENTRE FOR DISCRETE MATHEMATICS AND COMPUTING \\ DEPARTMENT OF MATHEMATICS \\ THE UNIVERSITY OF QUEENSLAND, 4072, AUSTRALIA
}

\begin{abstract}
A minimal defining set of a Steiner triple system on $v$ points $(S T S(v))$ is a partial Steiner triple system contained in only this $S T S(v)$, and such that any of its proper subsets is contained in at least two distinct $S T S(v)$ s. We consider the standard doubling and tripling constructions for $S T S(2 v+1)$ and $S T S(3 v)$ from $S T S(v)$ and show how minimal defining sets of an $S T S(v)$ gives rise to minimal defining sets in the larger systems. We use this to construct some new families of defining sets. For example, for Steiner triple systems on $3^{n}$ points, we construct minimal defining sets of volumes varying by as much as $7^{n-2}$.
\end{abstract}

\section{INTRODUCTION}

In this paper we focus our attention on certain partial Steiner triple systems, called minimal defining sets, which have the property that they are contained in precisely one Steiner triple system of the same order as the partial system and are minimal with respect to this property. We take the standard " $v$ to $2 v+1$ " and " $v$ to $3 v$ " constructions for Steiner triple systems and show how these constructions can be adapted to construct minimal defining sets. To facilitate the verification of the correctness of these constructions we represent the Steiner triple systems as latin squares. Known results in the corresponding theory for partial latin squares will aid our understanding of the theory in relation to partial Steiner triple systems.

Thus we begin this paper with the relevant definitions and background information. Then in Sections 3 and 4 we focus on minimal defining sets for Steiner triple systems and document, respectively, the " $v$ to $2 v+1$ " and " $v$ to $3 v$ " constructions. In Section 3 examples of minimal defining sets are found when the " $v$ to $2 v+1$ " construction is applied to both Steiner triple systems from projective geometries and Steiner triple systems not isomorphic to projective geometries. In Section 4 examples of minimal defining sets are found when the " $v$ to $3 v$ " construction is applied both to Steiner triple systems from affine geometries and to designs generated from the Hall triple system of order 81 .

\section{BACKGROUND INFORMATION}

A partial Steiner triple system $\operatorname{PSTS}(v),(V, \mathcal{B})$, is a set $V=\{0,1, \ldots, v-1\}$ and a collection $\mathcal{B}$ of 3 -subsets chosen from $V$ in such a way that each 2-subset of $V$ occurs in at most one of the 3-subsets. The 3-subsets are called blocks or triples. The order of the partial Steiner triple system is $v$ and the volume is $|\mathcal{B}|$. If the volume is $v(v-1) / 6$ then we say that the partial Steiner triple system is a Steiner triple system $S T S(v),(V, \mathcal{T})$, and we note that each of the 2-subsets of $V$ occurs in precisely one of the triples.

Research supported by the Australian Research Council A49937047, A49802044. 
A partial Steiner triple system, $(V, \mathcal{B})$, is said to be a defining set if there exists a unique Steiner triple system $(V, \mathcal{T})$ such that $\mathcal{B} \subseteq \mathcal{T}$. A $P S T S(v)(V, \mathcal{B})$ is said to be a minimal defining set if for any $B \in \mathcal{B}$ there exists at least one other Steiner triple system $(V, \mathcal{R})$ with $\mathcal{R}$ distinct from $\mathcal{T}$ such that $(\mathcal{B} \backslash\{B\}) \subseteq \mathcal{R}$. The set $\mathcal{T} \backslash(\mathcal{T} \cap \mathcal{R})$ is said to form a Steiner trade. In general if there exist two $\operatorname{PSTS}(v) \mathcal{E}$ and $\mathcal{E}^{\prime}$ such that $\mathcal{E} \cap \mathcal{E}^{\prime}=\emptyset,|\mathcal{E}|=\left|\mathcal{E}^{\prime}\right|$ and the 2-subset $\{a, b\}$ occurs in the triples of $\mathcal{E}$ if and only if it occurs in the triples of $\mathcal{E}^{\prime}$, then $\mathcal{E}$ is said to be a Steiner trade.

Throughout this paper $(V, \mathcal{B})$ will denote a partial Steiner triple system which is contained in a Steiner triple system $(V, \mathcal{T})$.

Example 1. Let $V=\{0,1,2, \ldots, 8\}$. Define

$$
\mathcal{T}=\left\{\begin{array}{llll}
\{0,1,2\}, & \{0,3,6\}, & \{0,4,8\}, & \{0,5,7\}, \\
\{3,4,5\}, & \{1,4,7\}, & \{1,5,6\}, & \{1,3,8\}, \\
\{6,7,8\}, & \{2,5,8\}, & \{2,3,7\}, & \{2,4,6\}
\end{array}\right\}
$$

and

$$
\mathcal{B}=\{\{0,1,2\}, \quad\{3,4,5\}, \quad\{0,3,6\}, \quad\{1,4,7\}\} .
$$

Then $(V, \mathcal{T})$ is an $S T S(9)$ and $(V, \mathcal{B})$ is a $\operatorname{PSTS}(9)$. Since $\mathcal{B}$ is a subset of $\mathcal{T}$ we say that $(V, \mathcal{B})$ is a partial Steiner triple system in $(V, \mathcal{T})$. Note that the partial Steiner triple system $(V, \mathcal{B})$ is actually a minimal defining set.

Lemma 2. (See, for example, [12].) Define

$$
\begin{aligned}
& \mathcal{E}_{0}=\{\{0,2,3\},\{0,4,5\},\{1,2,5\},\{1,3,4\}\}, \\
& \mathcal{E}_{1}=\{\{0,1,2\},\{0,4,8\},\{0,5,7\},\{6,1,5\},\{6,2,4\},\{6,7,8\}\}, \text { and } \\
& \mathcal{E}_{2}=\{\{0,3,4\},\{0,5,6\},\{1,3,5\},\{1,4,6\},\{2,3,6\},\{2,4,5\}\} .
\end{aligned}
$$

Then

(a) $\mathcal{E}_{0}$ is the unique Steiner trade of volume four, up to isomorphism, and

(b) $\mathcal{E}_{1}$ and $\mathcal{E}_{2}$ are the only two non-isomorphic Steiner trades of volume six.

In this paper $\mathcal{E}_{1}$ and $\mathcal{E}_{2}$ are called a type one trade and a type two trade, respectively. The trade $\mathcal{E}_{0}$ is crucial for the constructions given in Section 3 and the trade $\mathcal{E}_{1}$ is crucial for the constructions given in Section 4.

The identification of Steiner trades is vital to the identification of minimal defining sets. The next lemma clarifies the connection between minimal defining sets and trades.

Lemma 3. Take a Steiner triple system $(V, \mathcal{T})$ and a partial Steiner triple system $(V, \mathcal{B})$ such that $\mathcal{B} \subseteq \mathcal{T}$. Then $(V, \mathcal{B})$ is a minimal defining set if and only if:

1. (see [8]) for all trades $\mathcal{E} \subseteq \mathcal{T}, \mathcal{E} \cap \mathcal{B} \neq \emptyset$;

2. (see [9]) for each triple $B \in \mathcal{B}$, there exists a trade $\mathcal{E} \subseteq \mathcal{T}$ such that $\mathcal{E} \cap \mathcal{B}=\{B\}$.

Example 4. Let $(V, \mathcal{T})$ and $(V, \mathcal{B})$ be as in Example 1, and $\mathcal{E}_{1}$ as in Lemma 2. Define

$$
\mathcal{E}_{1}^{\prime}=\{\{0,1,5\},\{0,2,4\},\{0,7,8\},\{6,1,2\},\{6,4,8\},\{6,5,7\}\} .
$$

Then $\mathcal{E}_{1} \cap \mathcal{E}_{1}^{\prime}=\emptyset,\left|\mathcal{E}_{1}\right|=\left|\mathcal{E}_{1}^{\prime}\right|$ and each 2-subset occurring in the triples of $\mathcal{E}_{1}$ also occurs in the triples of $\mathcal{E}_{1}^{\prime}$. It is also worth noting that for the minimal defining set $\mathcal{B}$ given in Example $1, \mathcal{B} \backslash\{\{0,1,2\}\}$ is contained in a completion distinct from $\mathcal{T}$, namely $\left(\mathcal{T} \backslash \mathcal{E}_{1}\right) \cup \mathcal{E}_{1}^{\prime}$. Relevant Steiner trades can be found for each of the other triples of $\mathcal{B}$.

A partial latin square $P$ of order $v$ is a $v \times v$ array with entries chosen from the set $V=\{0,1, \ldots, v-1\}$ in such a way that each element of $V$ occurs at most once in each row and at most once in each column of the array. The size of the partial latin square is the number of non-empty cells in $P$. The notation $P(i, j)$ will be used to represent the 
occurrence of symbol $P(i, j) \in V$ in cell $(i, j)$ of the array. If each of the entries chosen from $V$ occurs exactly once in each row and once in each column, then the array $L$ is said to be a latin square, and $L(i, j)$ denotes the occurrence of symbol $L(i, j) \in V$ in cell $(i, j)$ of the array $L$.

\section{Example 5.}

\begin{tabular}{|l|l|l|l|l|l|l|l|l|l|}
\hline 9 & 2 & 1 & 6 & & & 3 & & & 0 \\
\hline 2 & 9 & 0 & & 7 & & & 4 & & 1 \\
\hline 1 & 0 & 9 & & & & & & & 2 \\
\hline 6 & & & 9 & 5 & 4 & 0 & & & 3 \\
\hline & 7 & & 5 & 9 & 3 & & 1 & & 4 \\
\hline & & & 4 & 3 & 9 & & & & 5 \\
\hline 3 & & & 0 & & & 9 & & & 6 \\
\hline & 4 & & & 1 & & & 9 & & 7 \\
\hline & & & & & & & & & \\
\hline 0 & 1 & 2 & 3 & 4 & 5 & 6 & 7 & & 9 \\
\hline
\end{tabular}

$P$ : Partial latin square of order 10

\begin{tabular}{|l|l|l|l|l|l|l|l|l|l|}
\hline 9 & 2 & 1 & 6 & 8 & 7 & 3 & 5 & 4 & 0 \\
\hline 2 & 9 & 0 & 8 & 7 & 6 & 5 & 4 & 3 & 1 \\
\hline 1 & 0 & 9 & 7 & 6 & 8 & 4 & 3 & 5 & 2 \\
\hline 6 & 8 & 7 & 9 & 5 & 4 & 0 & 2 & 1 & 3 \\
\hline 8 & 7 & 6 & 5 & 9 & 3 & 2 & 1 & 0 & 4 \\
\hline 7 & 6 & 8 & 4 & 3 & 9 & 1 & 0 & 2 & 5 \\
\hline 3 & 5 & 4 & 0 & 2 & 1 & 9 & 8 & 7 & 6 \\
\hline 5 & 4 & 3 & 2 & 1 & 0 & 8 & 9 & 6 & 7 \\
\hline 4 & 3 & 5 & 1 & 0 & 2 & 7 & 6 & 9 & 8 \\
\hline 0 & 1 & 2 & 3 & 4 & 5 & 6 & 7 & 8 & 9 \\
\hline \multicolumn{10}{|c|}{$L$}
\end{tabular}

Note that since the symbols in the non-empty cells of $P$ are identical to the symbols in the corresponding cells of $L$ we may think of $P$ as a subset of $L$. In general, if a latin square $L$ and a partial latin square $P$ are of the same order and if $P$ is a subset of $L$, then we say that $P$ is contained in $L$.

In the literature a minimal defining substructure for a latin square has been termed a critical set. So a critical set $C$ of order $v$ is a partial latin square which satisfies the properties:

1. $C$ is contained in precisely one latin square of order $v$;

2. for all $x \in C, C \backslash\{x\}$ is contained in at least two latin squares of order $v$.

We say that the partial latin square $C$ of order $v$ is uniquely completable to the latin square $L$ of order $v$ if $C$ satisfies condition 1 above.

Similarly, one may define a set of cells which form a trade within a latin square. That is, a partial latin square, $P$, forms a latin trade (sometimes called a latin interchange) if there exists a corresponding partial latin square, $P^{\prime}$, such that for all $i, j$, cell $(i, j)$ of $P$ contains $P(i, j) \in V$ if and only if cell $(i, j)$ of $P^{\prime}$ contains $P^{\prime}(i, j) \in V$ where $P(i, j) \neq P^{\prime}(i, j)$, and symbol $x \in V$ occurs in row (column) $i$ of $P$ if and only if $x$ occurs in row (column) $i$ of $P^{\prime}$. Once again there is a strong connection between critical sets and latin trades (see [11]).

Lemma 6. Let $L$ be a latin square of order $v$ and $C \subseteq L$. The partial latin square $C$ is a critical set if and only if:

- for all latin trades $I \subseteq L, I \cap C \neq \emptyset$;

- for all $x \in C$, there exists a latin trade $I \subseteq L$ such that $I \cap C=\{x\}$.

Steiner triple systems and partial Steiner triple systems can be represented as latin squares and partial latin squares in two different ways.

Definition 7. Let $(V, \mathcal{B})$ be a $P S T S(v)$, where $V=\{0,1,2, \ldots, v-1\}$. Then the partial sloop associated with $(V, \mathcal{B})$ is the partial latin square $P$ of order $v+1$ defined as follows:

1. for $0 \leq i, j \leq v-1, i \neq j$, the cell $(i, j)$ contains $k$ if and only if $\{i, j, k\} \in \mathcal{B}$;

2. the cell $(i, i)$ contains $v$ if $i$ occurs in a triple of $\mathcal{B}$;

3 . the cells $(i, v)$ and $(v, i)$ contain $i$ if $i$ occurs in a triple of $\mathcal{B}$;

4. the cell $(v, v)$ contains $v$.

Similarly if $(V, \mathcal{T})$ is an $S T S(v)$ there is a corresponding sloop associated with $(V, \mathcal{T})$. 
Example 5 provides an example of a sloop and a partial sloop of order 10 constructed, respectively, from the $S T S(9)$ and $P S T S(9)$ given in Example 1.

Definition 8. Let $(V, \mathcal{B})$ be a $P S T S(v)$, where $V=\{0,1,2, \ldots, v-1\}$. Then the partial Steiner latin square $P$ of order $v$ associated with $(V, \mathcal{B})$ is defined as follows:

1. for $0 \leq i, j \leq v-1, i \neq j$, the cell $(i, j)$ contains $k$ if and only if $\{i, j, k\} \in \mathcal{B}$;

2. the cell $(i, i)$ contains $i$ if and only if $i$ occurs in a triple of $\mathcal{B}$.

Similarly if $(V, \mathcal{T})$ is an $S T S(v)$ there is a corresponding Steiner latin square associated with $(V, \mathcal{T})$.

Note that in the definition of partial sloops and partial Steiner latin squares some elements of $V$ may not occur in any triple of $\mathcal{B}$ and therefore do not occur in the corresponding partial latin square.

The following lemma summarizes the connection between Steiner triple systems and sloops and Steiner latin squares. For Part 1 of the following lemma see [4]; Part 2 is clear by Definitions 7 and 8 .

Lemma 9. 1. The existence of a $\operatorname{PSTS}(v)$, a partial sloop of order $v+1$ and a partial Steiner latin square of order $v$ are all equivalent.

2. Let $(V, \mathcal{T})$ be an $S T S(v)$ and $(V, \mathcal{B})$ be a $P S T S(v)$, where $\mathcal{B} \subseteq \mathcal{T}$. Let $L$ be the sloop and $L^{\prime}$ the Steiner latin square associated with $(V, \mathcal{T})$, and $P$ be the partial sloop and $P^{\prime}$ the partial Steiner latin square associated with $(V, \mathcal{B})$. Then $P$ and $P^{\prime}$ are partial latin squares contained in $L$ and $L^{\prime}$ respectively.

\section{The $v$ To $2 v+1$ CONStRuCtion}

Construction 10. Let $(V, \mathcal{B})$ be a $\operatorname{PSTS}(v)$ and $(V, \mathcal{T})$ be an $S T S(v)$ where $V=\{0,1,2$, $\ldots, v-1\}$ and $\mathcal{B} \subseteq \mathcal{T}$. The doubling extension of $(V, \mathcal{B})$, denoted $\left(V^{*}, \mathcal{B}^{*}\right)$, is defined to be

$$
V^{*}=V \cup\{v, v+1, \ldots, 2 v\} \text { and } \mathcal{B}^{*}=\mathcal{B}_{1} \cup \mathcal{B}_{2} \cup \mathcal{B}_{3}
$$

where:

1. $\mathcal{B}_{1}=\{\{i, j, k\} \mid\{i, j, k\} \in \mathcal{T}\}$

2. $\mathcal{B}_{2}=\{\{i, j+v+1, k+v+1\},\{i+v+1, j, k+v+1\},\{i+v+1, j+v+1, k\} \mid$ $\{i, j, k\} \in \mathcal{B}$ and $i<j<k\}$;

3. $\mathcal{B}_{3}=\{\{i, v, i+v+1\} \mid i$ occurs in a triple of $\mathcal{B}\}$.

Similarly if $(V, \mathcal{T})$ is an $S T S(v)$, the doubling extension of $(V, \mathcal{T})$, will be denoted $\left(V^{*}, \mathcal{T}^{*}\right)$.

Lemma 11. (See [2] Theorem 0.1) Consider $\left(V^{*}, \mathcal{T}^{*}\right)$ and $\left(V^{*}, \mathcal{B}^{*}\right)$ as given in Construction 10. Then:

1. $\left(V^{*}, \mathcal{T}^{*}\right)$ is an $S T S(2 v+1)$;

2. $\left(V^{*}, \mathcal{B}^{*}\right)$ is a $\operatorname{PSTS}(2 v+1)$ in the Steiner triple system $\left(V^{*}, \mathcal{T}^{*}\right)$.

Example 12. Construction 10 can be applied to the $S T S(9)$ and $P S T S(9)$ given in Example 1 to obtain the following $S T S(19)$ and $P S T S(19)$ on the set $V=\{0,1,2, \ldots, 18\}$. The 32 triples above the line correspond to the $P S T S(19)$ and all 57 triples correspond to 
the $S T S(19)$.

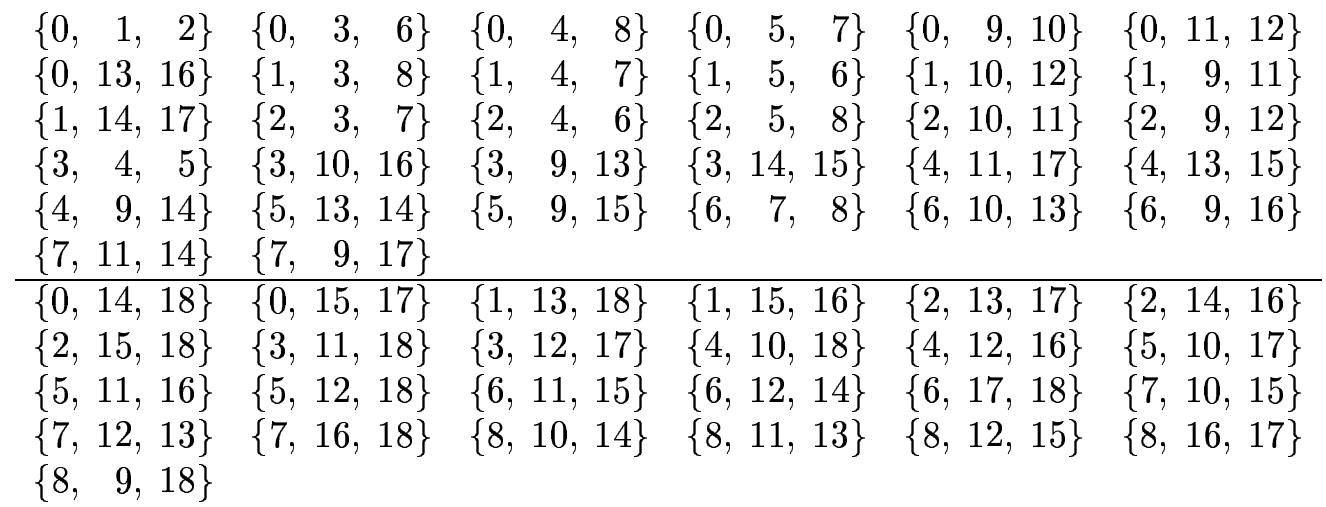

Construction 13. Let $P$ be a partial sloop of order $v+1$ on the set $\{0,1,2, \ldots, v\}$, contained in the sloop $L$ of order $v+1$. Define the partial latin square $\hat{P}$ and the latin square $\hat{L}$, of order $2 v+2$, by

$$
\hat{P}=\begin{array}{|l|l|}
\hline L^{0} & P^{1} \\
\hline P^{1} & P^{0} \\
\hline
\end{array} \quad \text { and } \hat{L}=\begin{array}{|l|l|}
\hline L^{0} & L^{1} \\
\hline L^{1} & L^{0} \\
\hline
\end{array}
$$

where $L^{0}$ and $P^{0}$ are copies of $L$ and $P$ respectively, as are $L^{1}$ and $P^{1}$ except that symbol $x$ is replaced by symbol $x+v+1$, for all $x \in\{0,1, \ldots, v\}$. Now the doubling extension of $P$, denoted $P^{*}$, is defined as follows.

1. $P^{*}(i, j)=\hat{P}(i, j)$ for $0 \leq i, j \leq 2 v+1, i \neq j$ and $i, j \notin\{v, 2 v+1\}$;

2. $P^{*}(i, v)=\hat{P}(i, 2 v+1)$ and $P^{*}(v, i)=\hat{P}(2 v+1, i)$ for $0 \leq i \leq 2 v+1$;

3. $P^{*}(i, 2 v+1)=\hat{P}(i, v)$ and $P^{*}(2 v+1, i)=\hat{P}(v, i)$ for $0 \leq i \leq 2 v+1$; and

4. $P^{*}(i, i)=2 v+1$ if $\hat{P}(i, i)=v$.

Note that $L^{*}$ is the analogue of $P^{*}$, with $\hat{L}$ defined as above.

Lemma 14. (See [6]) Let $L, \hat{L}, P$ and $\hat{P}$ be as in Construction 13. If $P$ is uniquely completable to $L$ then $\hat{P}$ is uniquely completable to $\hat{L}$.

Applying Lemma 14 one can prove the following lemma (see $[4,5])$.

Lemma 15. Let $L, P, L^{*}$ and $P^{*}$ be as given in Construction 13. Then:

1. $P^{*}$ is a partial sloop of order $2 v+2$ and $L^{*}$ is a sloop of order $2 v+2$;

2. $P^{*}$ is a partial latin square in $L^{*}$;

3. If $P$ is uniquely completable to $L$ then $P^{*}$ is uniquely completable to $L^{*}$.

Example 16. Consider $L$ and $P$ as in Example 5. By Construction 13 the doubling extension of $L$ is as follows. 


\begin{tabular}{|c|c|c|c|c|c|c|c|c|c||c|c|c|c|c|c|c|c|c|c|}
\hline 19 & 2 & 1 & 6 & 8 & 7 & 3 & 5 & 4 & 10 & 9 & 12 & 11 & 16 & 18 & 17 & 13 & 15 & 14 & 0 \\
\hline 2 & 19 & 0 & 8 & 7 & 6 & 5 & 4 & 3 & 11 & 12 & 9 & 10 & 18 & 17 & 16 & 15 & 14 & 13 & 1 \\
\hline 1 & 0 & 19 & 7 & 6 & 8 & 4 & 3 & 5 & 12 & 11 & 10 & 9 & 17 & 16 & 18 & 14 & 13 & 15 & 2 \\
\hline 6 & 8 & 7 & 19 & 5 & 4 & 0 & 2 & 1 & 13 & 16 & 18 & 17 & 9 & 15 & 14 & 10 & 12 & 11 & 3 \\
\hline 8 & 7 & 6 & 5 & 19 & 3 & 2 & 1 & 0 & 14 & 18 & 17 & 16 & 15 & 9 & 13 & 12 & 11 & 10 & 4 \\
\hline 7 & 6 & 8 & 4 & 3 & 19 & 1 & 0 & 2 & 15 & 17 & 16 & 18 & 14 & 13 & 9 & 11 & 10 & 12 & 5 \\
\hline 3 & 5 & 4 & 0 & 2 & 1 & 19 & 8 & 7 & 16 & 13 & 15 & 14 & 10 & 12 & 11 & 9 & 18 & 17 & 6 \\
\hline 5 & 4 & 3 & 2 & 1 & 0 & 8 & 19 & 6 & 17 & 15 & 14 & 13 & 12 & 11 & 10 & 18 & 9 & 16 & 7 \\
\hline 4 & 3 & 5 & 1 & 0 & 2 & 7 & 6 & 19 & 18 & 14 & 13 & 15 & 11 & 10 & 12 & 17 & 16 & 9 & 8 \\
\hline 10 & 11 & 12 & 13 & 14 & 15 & 16 & 17 & 18 & 19 & 0 & 1 & 2 & 3 & 4 & 5 & 6 & 7 & 8 & 9 \\
\hline \hline 9 & 12 & 11 & 16 & 18 & 17 & 13 & 15 & 14 & 0 & 19 & 2 & 1 & 6 & 8 & 7 & 3 & 5 & 4 & 10 \\
\hline 12 & 9 & 10 & 18 & 17 & 16 & 15 & 14 & 13 & 1 & 2 & 19 & 0 & 8 & 7 & 6 & 5 & 4 & 3 & 11 \\
\hline 11 & 10 & 9 & 17 & 16 & 18 & 14 & 13 & 15 & 2 & 1 & 0 & 19 & 7 & 6 & 8 & 4 & 3 & 5 & 12 \\
\hline 16 & 18 & 17 & 9 & 15 & 14 & 10 & 12 & 11 & 3 & 6 & 8 & 7 & 19 & 5 & 4 & 0 & 2 & 1 & 13 \\
\hline 18 & 17 & 16 & 15 & 9 & 13 & 12 & 11 & 10 & 4 & 8 & 7 & 6 & 5 & 19 & 3 & 2 & 1 & 0 & 14 \\
\hline 17 & 16 & 18 & 14 & 13 & 9 & 11 & 10 & 12 & 5 & 7 & 6 & 8 & 4 & 3 & 19 & 1 & 0 & 2 & 15 \\
\hline 13 & 15 & 14 & 10 & 12 & 11 & 9 & 18 & 17 & 6 & 3 & 5 & 4 & 0 & 2 & 1 & 19 & 8 & 7 & 16 \\
\hline 15 & 14 & 13 & 12 & 11 & 10 & 18 & 9 & 16 & 7 & 5 & 4 & 3 & 2 & 1 & 0 & 8 & 19 & 6 & 17 \\
\hline 14 & 13 & 15 & 11 & 10 & 12 & 17 & 16 & 9 & 8 & 4 & 3 & 5 & 1 & 0 & 2 & 7 & 6 & 19 & 18 \\
\hline 0 & 1 & 2 & 3 & 4 & 5 & 6 & 7 & 8 & 9 & 10 & 11 & 12 & 13 & 14 & 15 & 16 & 17 & 18 & 19 \\
\hline
\end{tabular}

By Construction 13 the doubling extension of $P$ is as follows.

\begin{tabular}{|c|c|c|c|c|c|c|c|c|c|c|c|c|c|c|c|c|c|c|}
\hline 19 & 2 & 1 & 6 & 8 & \begin{tabular}{|l|}
7 \\
\end{tabular} & 3 & 5 & 4 & 10 & 9 & 12 & 11 & \begin{tabular}{|l|}
16 \\
\end{tabular} & & & 13 & & 0 \\
\hline \begin{tabular}{l|}
2 \\
\end{tabular} & 19 & 0 & 8 & 7 & \begin{tabular}{l|l}
6 \\
\end{tabular} & 5 & 4 & 3 & 11 & 12 & 9 & 10 & & \begin{tabular}{|l|}
17 \\
\end{tabular} & & & 14 & $\overline{1}$ \\
\hline 1 & 0 & 19 & 7 & 6 & 8 & 4 & 3 & 5 & 12 & 11 & $\begin{array}{ll}10 \\
\end{array}$ & $\begin{array}{l}9 \\
\end{array}$ & & & & & & 2 \\
\hline 6 & 8 & 7 & 19 & 5 & 4 & 0 & 2 & 1 & 13 & 16 & & & \begin{tabular}{|l|}
9 \\
\end{tabular} & 15 & \begin{tabular}{|l|}
14 \\
\end{tabular} & 10 & & 3 \\
\hline 8 & 7 & 6 & 5 & $\begin{array}{l}19 \\
\end{array}$ & \begin{tabular}{|l|}
3 \\
\end{tabular} & 22 & $\overline{1}$ & 0 & 14 & & \begin{tabular}{|l|}
17 \\
\end{tabular} & & 15 & $\begin{array}{l}9 \\
\end{array}$ & \begin{tabular}{|l|l}
13 \\
\end{tabular} & & \begin{tabular}{|l|}
11 \\
\end{tabular} & 4 \\
\hline 7 & 6 & 8 & 4 & 3 & \begin{tabular}{|l|l}
19 \\
\end{tabular} & 1 & 0 & 2 & 15 & & & & 14 & 13 & 9 & & & 5 \\
\hline 3 & 5 & 4 & 0 & 2 & 1 & 19 & 8 & 7 & 16 & 13 & & & 10 & & & \begin{tabular}{l|}
9 \\
\end{tabular} & & 6 \\
\hline 5 & 4 & 3 & 2 & 1 & 0 & 8 & 19 & 6 & 17 & & 14 & & & 11 & & & \begin{tabular}{l|l|}
9 \\
\end{tabular} & 7 \\
\hline 4 & 3 & 5 & 1 & 0 & 2 & 7 & \begin{tabular}{l|l}
6 & \\
\end{tabular} & 19 & & & & & & & & & & 8 \\
\hline 10 & 11 & 12 & 13 & 14 & 15 & 16 & 17 & & 19 & 0 & 1 & 2 & 3 & 4 & 5 & 6 & 7 & 9 \\
\hline 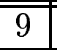 & $\bar{~} 12$ & $\bar{~} 11$ & $\bar{~} 16$ & & & 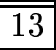 & & & 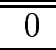 & 1819 & $\bar{~} 2$ & $\overline{c 1}$ & $\bar{~} 6$ & & & 3 & & $\overline{10}$ \\
\hline 12 & 9 & 10 & & 17 & & & 14 & & 1 & 2 & \begin{tabular}{|l|}
19 \\
\end{tabular} & 0 & & 7 & & & \begin{tabular}{|l|}
4 \\
\end{tabular} & 11 \\
\hline 11 & 10 & 9 & & & & & & & 2 & 1 & 0 & 19 & & & & & & 12 \\
\hline 16 & & & 9 & 15 & 14 & 10 & & & 3 & 6 & & & 19 & 5 & 4 & 0 & & 13 \\
\hline & 17 & & 15 & 9 & 13 & & 11 & & 4 & & 7 & & 5 & 19 & 3 & & 1 & 14 \\
\hline & & & 14 & 13 & \begin{tabular}{|l|}
9 \\
\end{tabular} & & & & 5 & & & & 4 & 3 & \begin{tabular}{|l|}
19 \\
\end{tabular} & & & 15 \\
\hline 13 & & & 10 & & & \begin{tabular}{|c|}
9 \\
\end{tabular} & & & 6 & 3 & & & 0 & & & 19 & & 16 \\
\hline & 14 & & & 11 & & & \begin{tabular}{l|}
9 \\
\end{tabular} & & 7 & & 4 & & & 1 & & & \begin{tabular}{|l|}
19 \\
\end{tabular} & 17 \\
\hline & & & & & & & & & & & & & & & & & & \\
\hline 0 & 1 & 2 & 3 & 4 & 5 & 6 & 7 & 8 & 9 & 10 & 11 & 12 & 13 & 14 & \begin{tabular}{|l|}
15 \\
\end{tabular} & 16 & \begin{tabular}{|l|}
17 \\
\end{tabular} & $\overline{19}$ \\
\hline
\end{tabular}

In addition we see that $L^{*}$ is the unique completion of $P^{*}$, illustrating Lemma 15.

Lemma 17 below states the relationship between the doubling extension of a (partial) Steiner triple system and the doubling extension of its corresponding (partial) sloop. We apply Definition 7 and Constructions 10 and 13 to prove this lemma.

Lemma 17. Let $(V, \mathcal{B})$ be a $P S T S(v)$ contained in the $S T S(v)(V, \mathcal{T})$. Moreover, let $P$ be the partial sloop corresponding to $(V, \mathcal{B})$ and let $L$ be the sloop corresponding to $(V, \mathcal{T})$. 
Then $P^{*}$ is the partial sloop corresponding to $\left(V^{*}, \mathcal{B}^{*}\right)$ and $L^{*}$ is the sloop corresponding to $\left(V^{*}, \mathcal{T}^{*}\right)$.

Proof. Let $P^{\prime}$ be the partial sloop associated with $\left(V^{*}, \mathcal{B}^{*}\right)$ and let $L^{\prime}$ be the sloop associated with $\left(V^{*}, \mathcal{T}^{*}\right)$ (see Definition 7 ). We need to show that $P^{\prime}=P^{*}$ and $L^{\prime}=L^{*}$. We only prove $P^{\prime}=P^{*}$. The other equality can be proved in a similar manner. Let $i, j, k \in V^{*}$ and $P^{\prime}(i, j)=k$. Since $P^{*}$ and $P^{\prime}$ are both symmetric we only prove $P^{*}(i, j)=P^{\prime}(i, j)$ for $i \leq j$.

Case 1: $i=j$. Then $P^{\prime}(i, i)=2 v+1$ and $i$ occurs in a triple of $\mathcal{B}^{*}$ by Definition 7 . If $i \in V$ then $i$ occurs in a triple of $\mathcal{B}$ by Construction 10 . So $P(i, i)=v$ by Definition 7 and $\hat{P}(i, i)=v$ by Construction 13. Therefore $P^{*}(i, i)=2 v+1$ by Construction 13. If $i=v$ then $P(i, i)=v$ by Definition 7 . So $\hat{P}(i, i)=v$ and $P^{*}(i, i)=2 v+1$ by Construction 13 . If $v+1 \leq i \leq 2 v+1$ then $i=i^{\prime}+v+1$ where $i^{\prime} \in V$ and $i^{\prime}$ occurs in a triple of $\mathcal{B}$. So $P\left(i^{\prime}, i^{\prime}\right)=v$ and $\hat{P}(i, i)=v$. Therefore $P^{*}(i, i)=2 v+1$ by Construction 13 .

Case 2: $i<j$. Let $P^{\prime}(i, j)=k$. If $i, j \in V$ then $\{i, j, k\} \in \mathcal{B}^{*}$ by Definition 7 and $\{i, j, k\} \in \mathcal{B}$ by Construction 10 . So we have $P(i, j)=k$. Now Construction 13 shows that $P^{*}(i, j)=\hat{P}(i, j)=P(i, j)=k$ as required.

The other subcases are $i \in V$ and $j=v, i \in V$ and $v+1 \leq j<2 v+1, i \in V$ and $j=2 v+1, i=v$ and $v+1 \leq j<2 v+1, i=v$ and $j=2 v+1$, and $v+1 \leq i<j<2 v+1$. In a similar fashion it can be shown that $P^{*}(i, j)=P^{\prime}(i, j)$ for each of these subcases.

One can check that the $S T S(19)$ and $P S T S(19)$ given in Example 12 correspond to the sloop and partial sloop given in Example 16.

To prove the main theorem of this section, Theorem 22, we make use of the following lemmas.

Lemma 18. Assume element $j$ does not occur in any triple of the partial Steiner triple system $(V, \mathcal{B})$. Then for the triple $\{i, v, i+v+1\}$ of $\mathcal{B}^{*}, i \in V$, there exists a trade $\mathcal{E}^{*}$ of volume four in $\mathcal{T}^{*}$ such that $\mathcal{E}^{*} \cap \mathcal{B}^{*}=\{\{i, v, i+v+1\}\}$.

Proof. Fix $i \in V$ and let $\{i, j, k\}$ be the triple of $\mathcal{T}$ which contains $\{i, j\}$. Since $j$ does not occur in any triple of $\mathcal{B}$ it follows that $\{i, j, k\}$ is not a triple of $\mathcal{B}$. Now define

$$
\mathcal{E}^{*}=\{\{i, v, i+v+1\},\{i, j+v+1, k+v+1\},\{j, v, j+v+1\},\{j, i+v+1, k+v+1\}\} .
$$

Then $\mathcal{E}^{*} \subseteq \mathcal{T}^{*}$ and $\mathcal{E}^{*} \cap \mathcal{B}^{*}=\{\{i, v, i+v+1\}\}$.

Lemma 19. Let $\{i, j, k\}$ be a triple in $\mathcal{T} \backslash \mathcal{B}$. Then there is a trade $\mathcal{E}^{*}$ of volume four in $\mathcal{T}^{*}$ such that $\mathcal{E}^{*} \cap \mathcal{B}^{*}=\{\{i, j, k\}\}$.

Proof. Define

$$
\mathcal{E}^{*}=\{\{i, j, k\},\{i, j+v+1, k+v+1\},\{i+v+1, j, k+v+1\},\{i+v+1, j+v+1, k\}\} .
$$

Then $\mathcal{E}^{*} \subseteq \mathcal{T}^{*}$ and $\mathcal{E}^{*} \cap \mathcal{B}^{*}=\{\{i, j, k\}\}$.

Lemma 20. Assume that for the triple $\{a, b, c\}$ of $\mathcal{B}$ there exists a trade

$$
\mathcal{E}=\{\{a, b, c\},\{a, d, e\},\{f, b, e\},\{f, c, d\}\}
$$

in $\mathcal{T}$ such that $\mathcal{E} \cap \mathcal{B}=\{\{a, b, c\}\}$. Then

(I) there exists a trade $\mathcal{E}^{*}$ in $\mathcal{T}^{*}$ of volume four such that $\mathcal{E}^{*} \cap \mathcal{B}^{*}=\{\{a, b+v+1, c+v+1\}\}$;

(II) there exists a trade $\mathcal{E}^{*}$ in $\mathcal{T}^{*}$ of volume four such that $\mathcal{E}^{*} \cap \mathcal{B}^{*}=\{\{a, b, c\}\}$.

Proof. (I) Define $\mathcal{E}^{*}$, the required trade, by

$\mathcal{E}^{*}=\{\{a, b+v+1, c+v+1\},\{a, d+v+1, e+v+1\},\{f, b+v+1, e+v+1\},\{f, c+v+1, d+v+1\}\}$. 
(II) Define $\mathcal{E}^{*}$, the required trade, by

$\mathcal{E}^{*}=\{\{a, b, c\},\{a, d+v+1, e+v+1\},\{f+v+1, b, e+v+1\},\{f+v+1, c, d+v+1\}\}$.

Lemma 21. (I) Assume that for the triple $\{a, b, c\}$ of $\mathcal{B}$ there exists a trade

$$
\mathcal{E}_{1}=\{\{a, b, c\},\{a, d, e\},\{a, f, g\},\{h, b, e\},\{h, d, g\},\{h, c, f\}\}
$$

in $\mathcal{T}$ such that $\mathcal{E}_{1} \cap \mathcal{B}=\{\{a, b, c\}\}$. Then there exists a trade $\mathcal{E}_{1}^{*}$ in $\mathcal{T}^{*}$ of type one such that $\mathcal{E}_{1}^{*} \cap \mathcal{B}^{*}=\{\{a, b+v+1, c+v+1\}\}$.

(II) Assume that for the triple $\{a, b, c\}$ of $\mathcal{B}$ there exists a trade

$$
\mathcal{E}_{2}=\{\{a, b, c\},\{a, d, e\},\{f, b, e\},\{f, c, d\},\{g, b, d\},\{g, c, e\}\}
$$

in $\mathcal{T}$ such that $\mathcal{E}_{2} \cap \mathcal{B}=\{\{a, b, c\}\}$. Then there exists a trade $\mathcal{E}_{2}^{*}$ in $\mathcal{T}^{*}$ of type two such that $\mathcal{E}_{2}^{*} \cap \mathcal{B}^{*}=\{\{a, b, c\}\}$.

Proof. (I) Define $\mathcal{E}_{1}^{*}$, the required trade, by

$$
\begin{aligned}
\mathcal{E}_{1}^{*}= & \{\{a, b+v+1, c+v+1\},\{a, d+v+1, e+v+1\},\{a, f+v+1, g+v+1\}, \\
& \{h, b+v+1, e+v+1\},\{h, d+v+1, g+v+1\},\{h, c+v+1, f+v+1\}\} .
\end{aligned}
$$

(II) Define $\mathcal{E}_{2}^{*}$, the required trade, by

$$
\begin{aligned}
\mathcal{E}_{2}^{*}= & \{\{a, b, c\},\{a, d+v+1, e+v+1\},\{f+v+1, b, e+v+1\}, \\
& \{f+v+1, c, d+v+1\},\{g+v+1, b, d+v+1\},\{g+v+1, c, e+v+1\}\} .
\end{aligned}
$$

Putting these results together leads us to the following theorem.

Theorem 22. Let $(V, \mathcal{T}),(V, \mathcal{B}), P,\left(V^{*}, \mathcal{T}^{*}\right),\left(V^{*}, \mathcal{B}^{*}\right)$ and $P^{*}$ be as before. Moreover, assume that:

(1) there exists only one element of $V$ which does not occur in any triple of $\mathcal{B}$;

(2) $P$ is uniquely completable to a latin square of order $v+1$;

(3) for each triple $\{i, j, k\}$ of $\mathcal{B}$ there is a trade $\mathcal{E}$ in $\mathcal{T}$ of volume four such that $\mathcal{E} \cap \mathcal{B}=$ $\{\{i, j, k\}\}$.

Then:

$\left(1^{\prime}\right)$ there exists only one element of $V^{*}$ which does not occur in any triple of $\mathcal{B}^{*}$;

$\left(2^{\prime}\right) P^{*}$ is uniquely completable to a latin square of order $2 v+2$;

$\left(3^{\prime}\right)$ for each triple $\{a, b, c\}$ of $\mathcal{B}^{*}$ there exists a trade $\mathcal{E}^{*}$ in $\mathcal{T}^{*}$ of volume four such that $\mathcal{E}^{*} \cap \mathcal{B}^{*}=\{\{a, b, c\}\}$.

Proof. By Construction 10 if the element $a+v+1$ does not occur in any triple of $\mathcal{B}^{*}$ then the element $a$ of $V$ does not occur in any triple of $\mathcal{B}$, thus proving $\left(1^{\prime}\right)$. Then $\left(2^{\prime}\right)$ follows by Lemma 15, Part 3, and ( $\left.3^{\prime}\right)$ follows by Lemmas 18, 19 and 20.

Remark 23. Consider $(V, \mathcal{B})$ as in Theorem 22 . Then $(V, \mathcal{B})$ is actually a minimal defining set in $(V, \mathcal{T})$ and Construction 10 shows that

$$
\left|\mathcal{B}^{*}\right|=v(v-1) / 6+3|\mathcal{B}|+(v-1)=\frac{(v-1)(v+6)}{6}+3|\mathcal{B}| .
$$

Now Theorem 22 and Remark 23 lead to the following corollary.

Corollary 24. Let $(V, \mathcal{T}),(V, \mathcal{B}), P,\left(V^{*}, \mathcal{T}^{*}\right),\left(V^{*}, \mathcal{B}^{*}\right)$ and $P^{*}$ be as in Theorem 22. Then $\left(V^{*}, \mathcal{B}^{*}\right)$ is a minimal defining set of order $2 v+1$ with $(v-1)(v+6) / 6+3|\mathcal{B}|$ triples. 
If we let $v=2^{n}-1$ then the above formula reduces to

$$
\left|\mathcal{B}^{*}\right|=\frac{1}{3} 2^{2 n-1}+2^{n-1}-\frac{5}{3}+3|\mathcal{B}| \text {. }
$$

So in the following lemma we use a recursive relation to develop a formula for the size of $\left|\mathcal{B}^{*}\right|$ when $v=2^{n}-1$.

Lemma 25. Let $m \geq 1$ be an integer and define a sequence as follows:

$$
\begin{aligned}
b(n+1) & =\frac{1}{3} 2^{2 n-1}+2^{n-1}-\frac{5}{3}+3 b(n), \text { and } \\
b(m) & =\beta .
\end{aligned}
$$

Then, for all $n \geq m$,

$$
b(n)=\left[\left(6 \beta-2^{2 m}+3.2^{m}-5\right) 3^{n-m}+2^{2 n}-3.2^{n}+5\right] / 6 .
$$

Proof. Substituting into $b(n+1)=\frac{1}{3} 2^{2 n-1}+2^{n-1}-\frac{5}{3}+3 b(n)$ gives

$$
\begin{aligned}
b(m+1) & =\frac{1}{3} 2^{2 m-1}+2^{m-1}-\frac{5}{3}+3 b(m) \\
& =\frac{2^{2 m}+3.2^{m}-10}{6}+3 \beta \\
& =\left[18 \beta-3.2^{2 m}+9.2^{m}-15+4.2^{2 m}-6.2^{m}+5\right] / 6 \\
& =\left[\left(6 \beta-2^{2 m}+3.2^{m}-5\right) 3^{m+1-m}+2^{2(m+1)}-3.2^{m+1}+5\right] / 6
\end{aligned}
$$

as required. Thus the statement is true for $n=m+1$. Assume the statement is true for $n=k \geq m$ and we will prove it is true for $n=k+1$. That is, we will prove

$$
b(k+1)=\left[\left(6 \beta-2^{2 m}+3.2^{m}-5\right) 3^{k+1-m}+2^{2(k+1)}-3.2^{k+1}+5\right] / 6 .
$$

We know

$$
b(k+1)=\frac{1}{3} 2^{2 k-1}+2^{k-1}-\frac{5}{3}+3 b(k)
$$

and so by the inductive hypothesis

$$
\begin{aligned}
b(k+1) & =\frac{1}{3} 2^{2 k-1}+2^{k-1}-\frac{5}{3}+3\left[\left(6 \beta-2^{2 m}+3.2^{m}-5\right) 3^{k-m}+2^{2 k}-3.2^{k}+5\right] / 6 \\
& =\left[\left(6 \beta-2^{2 m}+3.2^{m}-5\right) 3^{k+1-m}+2^{2(k+1)}-3.2^{k+1}+5\right] / 6
\end{aligned}
$$

as required.

Corollary 26. If there exists a minimal defining set of order $2^{m}-1$, where $m \geq 2$ with volume $\beta$, then for all $n \geq m$ there exists a minimal defining set of order $2^{n}-1$ containing $b(n)$ triples where

$$
b(n)=\left[\left(6 \beta-2^{2 m}+3.2^{m}-5\right) 3^{n-m}+2^{2 n}-3.2^{n}+5\right] / 6 .
$$

\subsection{Applications of the Doubling CONSTRUCtion}

A Steiner triple system of order $2^{n+1}-1, n \geq 2$, can be obtained as follows from a projective geometry $P G(n, 2)$. Let $V$ be a $(n+1)$-dimensional vector space, $n \geq 2$, over $G F(2)$, the Galois field of order 2. Define

$$
\mathcal{T}=\{\{x, y, z\} \mid x, y, z \in V \backslash\{0\} \text { and } x+y+z=0\} .
$$

Then $(V, \mathcal{T})$ is a Steiner triple system of order $2^{n+1}-1$.

The following result was first obtained by Gower [5] in 1993. Her proof was based on finite geometry and is quite different from our proof. 
Corollary 27. There exists a minimal defining set, having

$$
\left[4^{n+1}-3^{n+1}-3.2^{n+1}+5\right] / 6
$$

triples, in the Steiner triple system of order $2^{n+1}-1, n \geq 2$, obtained from $P G(n, 2)$.

Proof. When $n=2$ the Steiner triple system $(V, \mathcal{T})$ obtained from $P G(2,2)$ is the unique $S T S(7)$. Let $V=\{0,1,2, \ldots, 6\}$,

$$
\mathcal{T}=\{\{0,1,3\},\{1,2,4\},\{2,3,5\},\{3,4,6\},\{4,5,0\},\{5,6,1\},\{6,0,2\}\}
$$

and $\mathcal{B}=\{\{0,1,3\},\{1,2,4\},\{2,3,5\}\}$. Then $(V, \mathcal{B})$ is a $P S T S(7)$ in $(V, \mathcal{T})$. The corresponding sloop of order 8 and the partial sloop of order 8 are shown below.

\begin{tabular}{|l|l|l|l|l|l|l|l|}
\hline 7 & 3 & 6 & 1 & 5 & 4 & 2 & 0 \\
\hline 3 & 7 & 4 & 0 & 2 & 6 & 5 & 1 \\
\hline 6 & 4 & 7 & 5 & 1 & 3 & 0 & 2 \\
\hline 1 & 0 & 5 & 7 & 6 & 2 & 4 & 3 \\
\hline 5 & 2 & 1 & 6 & 7 & 0 & 3 & 4 \\
\hline 4 & 6 & 3 & 2 & 0 & 7 & 1 & 5 \\
\hline 2 & 5 & 0 & 4 & 3 & 1 & 7 & 6 \\
\hline 0 & 1 & 2 & 3 & 4 & 5 & 6 & 7 \\
\hline \multicolumn{7}{|c|}{$L: 5 l o p$ of order 8}
\end{tabular}

\begin{tabular}{|l|l|l|l|l|l|l|l|}
\hline 7 & 3 & & 1 & & & & 0 \\
\hline 3 & 7 & 4 & 0 & 2 & & & 1 \\
\hline & 4 & 7 & 5 & 1 & 3 & & 2 \\
\hline 1 & 0 & 5 & 7 & & 2 & & 3 \\
\hline & 2 & 1 & & 7 & & & 4 \\
\hline & & 3 & 2 & & 7 & & 5 \\
\hline & & & & & & & \\
\hline 0 & 1 & 2 & 3 & 4 & 5 & & 7 \\
\hline
\end{tabular}

As Gower noted in [5] if we start the recursive construction explained in Construction 13 with $L$ above, then the Steiner triple systems corresponding to these sloops are isomorphic to the Steiner triple systems obtained from a projective geometry. On the other hand, $\mathcal{B}$ and $P$ satisfy Conditions (1), (2) and (3) in Theorem 22. Now the result follows by Corollary 24 and Corollary 26.

We conclude this section with two examples which satisfy Conditions (1), (2) and (3) in Theorem 22. With these examples we verify the existence of new families of minimal defining sets.

Example 28. The following 35 triples form an $S T S(15)$ on the element set $\{0,1,2, \ldots, 14\}$ and the 17 triples above the line form a minimal defining set in this $S T S(15)$.

\begin{tabular}{rrrrrrr}
$\{0,1,13\}$ & $\{0,2,6\}$ & $\{0,7,8\}$ & $\{0,9,11\}$ & $\{1,8,11\}$ & $\{1,7,9\}$ \\
$\{1,10,12\}$ & $\{2,9,12\}$ & $\{2,7,10\}$ & $\{2,11,13\}$ & $\{3,4,6\}$ & $\{3,8,9\}$ \\
$\{3,10,13\}$ & $\{4,9,10\}$ & $\{4,7,12\}$ & $\{5,10,11\}$ & $\{5,7,13\}$ & \\
\hline$\{0,3,12\}$ & $\{0,4,5\}$ & $\{0,10,14\}$ & $\{1,2,4\}$ & $\{1,3,14\}$ & $\{1,5,6\}$ \\
$\{12,13,14\}$ & $\{2,3,5\}$ & $\{2,8,14\}$ & $\{3,7,11\}$ & $\{4,8,13\}$ & $\{4,11,14\}$ \\
$\{5,8,12\}$ & $\{5,9,14\}$ & $\{6,7,14\}$ & $\{6,8,10\}$ & $\{6,9,13\}$ & $\{6,11,12\}$
\end{tabular}

Note that this design is not isomorphic to the $S T S(15)$ obtained from $P G(3,2)$. Also the element 14 does not occur in any triple of the minimal defining set. A computer search shows that for each of these 17 triples in the minimal defining set, there exists a trade of volume four which intersects the minimal defining set in only that triple.

Here is the partial sloop corresponding to this minimal defining set. It is a critical set and so has a unique completion. 


\begin{tabular}{|c|c|c|c|c|c|c|c|c|c|c|c|c|c|c|c|}
\hline 15 & 13 & 6 & & & & 2 & 8 & 7 & 11 & & 9 & & 1 & & 0 \\
\hline 13 & 15 & & & & & & 9 & 11 & 7 & 12 & 8 & 10 & 0 & & 1 \\
\hline 6 & & 15 & & & & 0 & 10 & & 12 & 7 & 13 & 9 & 11 & & 2 \\
\hline & & & 15 & 6 & & 4 & & 9 & 8 & 13 & & & 10 & & 3 \\
\hline & & & 6 & 15 & & 3 & 12 & & 10 & 9 & & 7 & & & 4 \\
\hline & & & & & 15 & & 13 & & & 11 & 10 & & 7 & & 5 \\
\hline 2 & & 0 & 4 & 3 & & 15 & & & & & & & & & 6 \\
\hline 8 & 9 & 10 & & 12 & 13 & & 15 & 0 & 1 & 2 & & 4 & 5 & & 7 \\
\hline 7 & 11 & & 9 & & & & 0 & 15 & 3 & & 1 & & & & 8 \\
\hline 11 & 7 & 12 & 8 & 10 & & & 1 & 3 & 15 & 4 & 0 & 2 & & & 9 \\
\hline & 12 & 7 & 13 & 9 & 11 & & 2 & & 4 & 15 & 5 & 1 & 3 & & 10 \\
\hline 9 & 8 & 13 & & & 10 & & & 1 & 0 & 5 & 15 & & 2 & & 11 \\
\hline & 10 & 9 & & 7 & & & 4 & & 2 & 1 & & 15 & & & 12 \\
\hline 1 & 0 & 11 & 10 & & 7 & & 5 & & & 3 & 2 & & 15 & & 13 \\
\hline & & & & & & & & & & & & & & & \\
\hline 0 & 1 & 2 & 3 & 4 & 5 & 6 & 7 & 8 & 9 & 10 & 11 & 12 & 13 & & 15 \\
\hline
\end{tabular}

Corollary 29. For all $n \geq 4$, there exists a minimal defining set of order $2^{n}-1$ containing $b(n)$ triples where

$$
b(n)=\left(\frac{17}{81}-\frac{71}{162}\right) 3^{n}+\frac{2^{2 n}-3.2^{n}+5}{6} .
$$

Example 30. The following 35 triples form an $S T S(15)$ on the element set $\{0,1,2, \ldots, 14\}$. The 21 triples above the line form a minimal defining set for this $S T S(15)$.

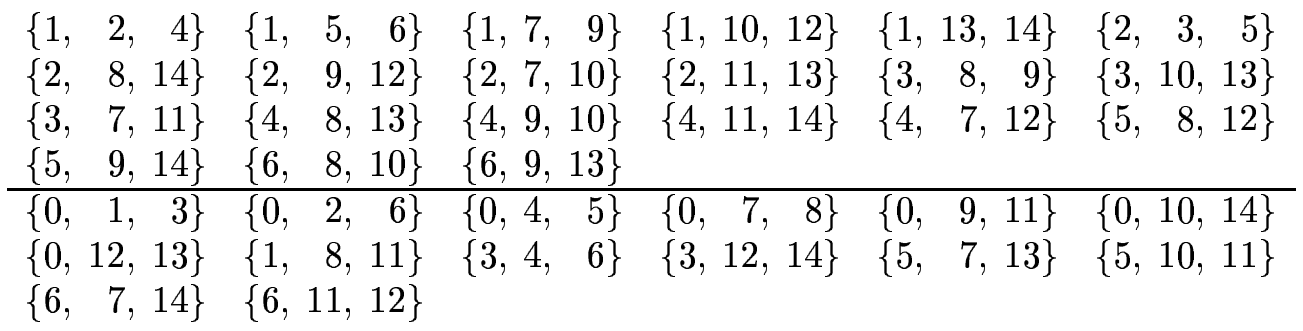

Note that this design is isomorphic to the $S T S(15)$ obtained from $P G(3,2)$. Also note that the element zero does not occur in any triple. A computer search shows that for each of these 21 triples of the minimal defining set there exists a trade of volume four which intersects the minimal defining set in only that triple.

Here is the partial sloop corresponding to this minimal defining set. It is a critical set and so has a unique completion. 


\begin{tabular}{|c|c|c|c|c|c|c|c|c|c|c|c|c|c|c|c|}
\hline & & & & & & & & & & & & & & & \\
\hline & 15 & 4 & & 2 & 6 & 5 & 9 & & 7 & 12 & & 10 & 14 & 13 & 1 \\
\hline & 4 & 15 & 5 & 1 & 3 & & 10 & 14 & 12 & 7 & 13 & 9 & 11 & 8 & 2 \\
\hline & & 5 & 15 & & 2 & & 11 & 9 & 8 & 13 & 7 & & 10 & & 3 \\
\hline & 2 & 1 & & 15 & & & 12 & 13 & 10 & 9 & 14 & 7 & 8 & 11 & 4 \\
\hline & 6 & 3 & 2 & & 15 & 1 & & 12 & 14 & & & 8 & & 9 & 5 \\
\hline & 5 & & & & 1 & 15 & & 10 & 13 & 8 & & & 9 & & 6 \\
\hline & 9 & 10 & 11 & 12 & & & 15 & & 1 & 2 & 3 & 4 & & & 7 \\
\hline & & 14 & 9 & 13 & 12 & 10 & & 15 & 3 & 6 & & 5 & 4 & 2 & 8 \\
\hline & 7 & 12 & 8 & 10 & 14 & 13 & 1 & 3 & 15 & 4 & & 2 & 6 & 5 & 9 \\
\hline & 12 & 7 & 13 & 9 & & 8 & 2 & 6 & 4 & 15 & & 1 & 3 & & 10 \\
\hline & & 13 & 7 & 14 & & & 3 & & & & 15 & & 2 & 4 & 11 \\
\hline & 10 & 9 & & 7 & 8 & & 4 & 5 & 2 & 1 & & 15 & & & 12 \\
\hline & 14 & 11 & 10 & 8 & & 9 & & 4 & 6 & 3 & 2 & & 15 & 1 & 13 \\
\hline & 13 & 8 & & 11 & 9 & & & 2 & 5 & & 4 & & 1 & 15 & 14 \\
\hline & 1 & 2 & 3 & 4 & 5 & 6 & 7 & 8 & 9 & 10 & 11 & 12 & 13 & 14 & 15 \\
\hline
\end{tabular}

Corollary 31. For all $n \geq 4$, there exists a minimal defining set of order $2^{n}-1$ containing $b(n)$ triples where

$$
b(n)=\left(\frac{21}{81}-\frac{71}{162}\right) 3^{n}+\frac{2^{2 n}-3.2^{n}+5}{6} .
$$

\section{THE $v$ TO $3 v$ CONSTRUCTION}

Construction 32. Let $(V, \mathcal{B})$ be a $\operatorname{PSTS}(v)$ and $(V, \mathcal{T})$ be an $S T S(v)$, where $V=\{0,1,2$, $\ldots, v-1\}$ and $\mathcal{B} \subseteq \mathcal{T}$. The tripling extension of a partial Steiner triple system, $(V, \mathcal{B})$, denoted $\left(V^{\bullet}, \mathcal{B}^{\bullet}\right)$, is defined to be

$$
V^{\bullet}=V \cup\{v, v+1, \ldots, 3 v-1\} \text { and } \mathcal{B}^{\bullet}=\mathcal{B}_{1} \cup \mathcal{B}_{2} \cup \mathcal{B}_{3} \cup \mathcal{B}_{4} \cup \mathcal{B}_{5}
$$

where:

1. $\mathcal{B}_{1}=\{\{i, j, k\} \mid\{i, j, k\} \in \mathcal{T}\}$;

2. $\mathcal{B}_{2}=\{\{i+v, j+v, k+v\} \mid\{i, j, k\} \in \mathcal{T}\}$

3. $\mathcal{B}_{3}=\{\{i+2 v, j+2 v, k+2 v\} \mid\{i, j, k\} \in \mathcal{B}\}$;

4. $\mathcal{B}_{4}=\{\{i, j+v, k+2 v\},\{i, j+2 v, k+v\},\{i+v, j, k+2 v\},\{i+v, j+2 v, k\}$,

$\{i+2 v, j, k+v\},\{i+2 v, j+v, k\} \mid\{i, j, k\} \in \mathcal{B}$ and $i<j<k\}$;

5. $\mathcal{B}_{5}=\{\{i, i+v, i+2 v\} \mid i$ occurs in a triple of $\mathcal{B}\}$.

¿From now on the tripling extension of the $S T S(v),(V, \mathcal{T})$, will be denoted $\left(V^{\bullet}, \mathcal{T}^{\bullet}\right)$.

The following lemma verifies that the designs obtained in Construction 32 are $S T S(3 v)$ and $P S T S(3 v)$, respectively.

Lemma 33. (See [2] Chapter 3) Consider $\left(V^{\bullet}, \mathcal{T}^{\bullet}\right)$ and $\left(V^{\bullet}, \mathcal{B}^{\bullet}\right)$ as in Construction 32. Then:

1. $\left(V^{\bullet}, \mathcal{T}^{\bullet}\right)$ is an $S T S(3 v)$;

2. $\left(V^{\bullet}, \mathcal{B}^{\bullet}\right)$ is a $\operatorname{PSTS}(3 v)$ in the Steiner triple system $\left(V^{\bullet}, \mathcal{T}^{\bullet}\right)$.

Now we state the relationship between the partial Steiner latin square associated with a partial Steiner triple system (see Definition 8) and the partial Steiner latin square associated with its tripling extension. The proof is straightforward and we leave it to the reader.

Lemma 34. Let $(V, \mathcal{T})$ be a Steiner triple system of order $v$ and let $L$ be the Steiner latin square associated with $(V, \mathcal{T})$. Let $(V, \mathcal{B})$ be a partial Steiner triple system contained in $(V, \mathcal{T})$ and let $P$ be the partial Steiner latin square associated with $(V, \mathcal{B})$. The partial Steiner latin square associated with $\left(V^{\bullet}, \mathcal{B}^{\bullet}\right)$ is $P^{\bullet}$, where for all $0 \leq i, j \leq v-1$ : 
1. $P^{\bullet}(i, j)=L(i, j), P^{\bullet}(i+v, j+2 v)=P^{\bullet}(i+2 v, j+v)=P(i, j)$;

2. $P^{\bullet}(i+v, j+v)=L(i, j)+v, P^{\bullet}(i, j+2 v)=P^{\bullet}(i+2 v, j)=P(i, j)+v$;

3. $P^{\bullet}(i, j+v)=P^{\bullet}(i+v, j)=P^{\bullet}(i+2 v, j+2 v)=P(i, j)+2 v$.

We define the Steiner latin square, $L^{\bullet}$, associated with $\left(V^{\bullet}, \mathcal{T}^{\bullet}\right)$ in a similar fashion.

The latin square $L^{\bullet}$ and the partial latin square $P^{\bullet}$ may be represented as arrays that are partitioned as follows. Note $L^{0}$ and $P^{0}$ are identical copies of $L$ and $P$ respectively, while $L^{1}$ and $P^{1}$ and $L^{2}$ and $P^{2}$ are copies of $L$ and $P$ but with symbol $x$ replaced by symbol $x+v$ and $x+2 v$ respectively.

$$
L^{\bullet}=\begin{array}{|l|l|l|}
\hline L^{0} & L^{2} & L^{1} \\
\hline L^{2} & L^{1} & L^{0} \\
\hline L^{1} & L^{0} & L^{2} \\
\hline
\end{array} \quad \text { and } P^{\bullet}=\begin{array}{|l|l|l|}
\hline L^{0} & P^{2} & P^{1} \\
\hline P^{2} & L^{1} & P^{0} \\
\hline P^{1} & P^{0} & P^{2} \\
\hline
\end{array}
$$

Lemma 35. (See [6]) Let $L, L^{\bullet}, P$ and $P^{\bullet}$ be as in Lemma 34. If $P$ is uniquely completable to $L$ then $P^{\bullet}$ is uniquely completable to $L^{\bullet}$.

To prove the main theorem of this section, Theorem 42 , we make use of the following lemmas.

Lemma 36. Assume element $j$ does not occur in any triple of the partial Steiner triple system $(V, \mathcal{B})$. Then for a triple $\{i, i+v, i+2 v\}$ of $\mathcal{B}^{\bullet}$ there is a trade $\mathcal{E}^{\bullet}$ of type one in $\mathcal{T}^{\bullet}$ such that $\mathcal{E}^{\bullet} \cap \mathcal{B}^{\bullet}=\{\{i, i+v, i+2 v\}\}$.

Proof. Fix $i \in V$ and let $\{i, j, k\}$ be the triple of $\mathcal{T}$ which contains the pair $\{i, j\}$. Since $j$ does not occur in any triple of $\mathcal{B}$ we have $\{i, j, k\} \notin \mathcal{B}$. Now define $\mathcal{E}^{\bullet}$, the required trade, to be

$$
\begin{aligned}
\mathcal{E}^{\bullet}= & \{\{i, i+v, i+2 v\},\{i, j+2 v, k+v\},\{i, j+v, k+2 v\}, \\
& \{j, j+v, j+2 v\},\{j, i+v, k+2 v\},\{j, i+2 v, k+v\}\} .
\end{aligned}
$$

Lemma 37. Let $\{i, j, k\}$ be a triple in $\mathcal{B}_{1} \backslash \mathcal{B}$ (see Construction 32 for a definition of $\mathcal{B}_{1}$ ). Then there is a trade $\mathcal{E}^{\bullet}$ of type one in $\mathcal{T}^{\bullet}$ such that $\mathcal{E}^{\bullet} \cap \mathcal{B}^{\bullet}=\{\{i, j, k\}\}$.

Proof. Define $\mathcal{E}^{\bullet}$, the required trade, to be

$$
\begin{aligned}
\mathcal{E}^{\bullet}= & \{\{i, j, k\},\{i, j+v, k+2 v\},\{i, j+2 v, k+v\}, \\
& \{i+2 v, j+2 v, k+2 v\},\{i+2 v, j+v, k\},\{i+2 v, j, k+v\}\} .
\end{aligned}
$$

Lemma 38. Let $\{i+v, j+v, k+v\}$ be a triple in $\mathcal{B}_{2} \backslash \mathcal{B}$ (see Construction 32 for a definition of $\mathcal{B}_{2}$ ). Then there is a trade $\mathcal{E}^{\bullet}$ of type one in $\mathcal{T}^{\bullet}$ such that $\mathcal{E}^{\bullet} \cap \mathcal{B}^{\bullet}=\{\{i+v, j+v, k+v\}\}$.

Proof. Define $\mathcal{E}^{\bullet}$, the required trade, to be

$$
\begin{aligned}
\mathcal{E}^{\bullet}= & \{\{i+v, j+v, k+v\},\{i+v, j, k+2 v\},\{i+v, j+2 v, k\}, \\
& \{i+2 v, j+2 v, k+2 v\},\{i+2 v, j, k+v\},\{i+2 v, j+v, k\}\} .
\end{aligned}
$$

The proof of the following lemma is straightforward and we leave it to the reader.

Lemma 39. Let $\{i, j, k\}$ be a triple in $\mathcal{B}$ and let $\mathcal{E}$ be a trade in $\mathcal{T}$ such that $\mathcal{E} \cap \mathcal{B}=$ $\{\{i, j, k\}\}$. Then there exists a trade $\mathcal{E}^{\bullet}$, isomorphic to $\mathcal{E}$, in $\mathcal{T}^{\bullet}$ such that $\mathcal{E}^{\bullet} \cap \mathcal{B}^{\bullet}=$ $\{\{i+2 v, j+2 v, k+2 v\}\}$. 
Lemma 40. Let $\{a, b, c\}$ be a triple of $\mathcal{B}$ and let

$$
\mathcal{E}=\{\{a, b, c\},\{a, d, e\},\{a, f, g\},\{h, d, g\},\{h, b, e\},\{h, f, c\}\}
$$

be a trade in $\mathcal{T}$ such that $\mathcal{E} \cap \mathcal{B}=\{\{a, b, c\}\}$. Then there exist trades $\mathcal{E}_{0}^{\bullet}$ and $\mathcal{E}_{1}^{\bullet}$, of type one, in $\mathcal{T}^{\bullet}$ such that $\mathcal{E}_{0}^{\bullet} \cap \mathcal{B}^{\bullet}=\{\{a, b, c\}\}$ and $\mathcal{E}_{1}^{\bullet} \cap \mathcal{B}^{\bullet}=\{\{a+v, b+v, c+v\}\}$.

Proof. First define

$$
\begin{aligned}
\mathcal{E}_{0}^{\bullet}= & \{\{a, b, c\},\{a, d+2 v, e+v\},\{a, f+v, g+2 v\}, \\
& \{h+2 v, d+2 v, g+2 v\},\{h+2 v, b, e+v\},\{h+2 v, f+v, c\}\} .
\end{aligned}
$$

By Construction 32 we have $\mathcal{E}_{0}^{\bullet} \subseteq \mathcal{T}^{\bullet}$. Now the fact that $\mathcal{E} \cap \mathcal{B}=\{\{a, b, c\}\}$ leads to $\mathcal{E}_{0}^{\bullet} \cap \mathcal{B}^{\bullet}=\{\{a, b, c\}\}$. Next define

$$
\begin{aligned}
\mathcal{E}_{1}^{\bullet}= & \{\{a+v, b+v, c+v\},\{a+v, d+2 v, e\},\{a+v, f, g+2 v\}, \\
& \{h+2 v, d+2 v, g+2 v\},\{h+2 v, e, b+v\},\{h+2 v, f, c+v\}\} .
\end{aligned}
$$

Similarly, the triples of $\mathcal{E}_{1}^{\bullet}$ are in $\mathcal{T}^{\bullet}$ and $\mathcal{E}_{1}^{\bullet} \cap \mathcal{B}^{\bullet}=\{\{a+v, b+v, c+v\}\}$.

Lemma 41. Let $\{a, b, c\}$ be a triple of $\mathcal{B}$ and let

$$
\mathcal{E}=\{\{a, b, c\},\{a, d, e\},\{a, f, g\},\{h, d, g\},\{h, b, e\},\{h, f, c\}\}
$$

be a trade in $\mathcal{T}$ such that $\mathcal{E} \cap \mathcal{B}=\{\{a, b, c\}\}$. Then there exists a trade $\mathcal{E}^{\bullet}$, of type one, in $\mathcal{T}^{\bullet}$ such that $\mathcal{E}^{\bullet} \cap \mathcal{B}^{\bullet}=\{\{a, b+v, c+2 v\}\}$.

Proof. Define

$$
\begin{aligned}
\mathcal{E}^{\bullet}= & \{\{a, b+v, c+2 v\},\{a, d+v, e+2 v\},\{a, f+v, g+2 v\}, \\
& \{h, d+v, g+2 v\},\{h, b+v, e+2 v\}\},\{h, f+v, c+2 v\}\} .
\end{aligned}
$$

Then by Construction 32 we have $\mathcal{E}^{\bullet} \subseteq \mathcal{T}^{\bullet}$. Moreover, the fact that $\mathcal{E} \cap \mathcal{B}=\{\{a, b, c\}\}$ leads to $\mathcal{E}^{\bullet} \cap \mathcal{B}^{\bullet}=\{\{a, b+v, c+2 v\}\}$.

Putting these results together leads us to the following theorem.

Theorem 42. Let $(V, \mathcal{T}),(V, \mathcal{B}), P,\left(V^{\bullet}, \mathcal{T}^{\bullet}\right),\left(V^{\bullet}, \mathcal{B}^{\bullet}\right)$ and $P^{\bullet}$ be as before. Moreover, assume that:

(1) there exists only one element of $V$ which does not occur in any triple of $\mathcal{B}$;

(2) $P$ is uniquely completable to a latin square of order $v$;

(3) for each triple $\{i, j, k\}$ of $\mathcal{B}$ there is a trade $\mathcal{E}$ in $\mathcal{T}$, of type one, such that $\mathcal{E} \cap \mathcal{B}=$ $\{\{i, j, k\}\}$.

Then:

$\left(1^{\prime}\right)$ there exists only one element of $V^{\bullet}$ which does not occur in any triple of $\mathcal{B}^{\bullet}$;

$\left(2^{\prime}\right) P^{\bullet}$ is uniquely completable to a latin square of order $3 v$;

$\left(3^{\prime}\right)$ for each triple $\{a, b, c\}$ of $\mathcal{B}^{\bullet}$ there exists a trade $\mathcal{E}^{\bullet}$ in $\mathcal{T}^{\bullet}$ of type one such that $\mathcal{E}^{\bullet} \cap \mathcal{B}^{\bullet}=\{\{a, b, c\}\}$.

Proof. By Construction 32, if the element $a+2 v$ does not occur in any triple of $\mathcal{B}^{\bullet}$ then the element $a$ of $V$ does not occur in any triple of $\mathcal{B}$, proving $\left(1^{\prime}\right)$. Then $\left(2^{\prime}\right)$ follows by Lemma 35 and $\left(3^{\prime}\right)$ follows by Lemmas $41,40,39,38$ and 37.

Remark 43. Consider $(V, \mathcal{B})$ as in Theorem 42 . Then $(V, \mathcal{B})$ is actually a minimal defining set in $(V, \mathcal{T})$, and Construction 32 shows that

$$
\left|\mathcal{B}^{\bullet}\right|=2\left(\frac{v(v-1)}{6}\right)+7|\mathcal{B}|+(v-1)=\frac{(v-1)(2 v+6)}{6}+7|\mathcal{B}| .
$$

Now Theorem 42 and Remark 43 lead to the following corollary. 
Corollary 44. Let $(V, \mathcal{T}),(V, \mathcal{B}), P,\left(V^{\bullet}, \mathcal{T}^{\bullet}\right),\left(V^{\bullet}, \mathcal{B}^{\bullet}\right)$ and $P^{\bullet}$ be as in Theorem 42. Then $\left(V^{\bullet}, \mathcal{B}^{\bullet}\right)$ is a minimal defining set of order $3 v$ with $(v-1)(2 v+6) / 6+7|\mathcal{B}|$ triples.

If we let $v=3^{n}$, then the above formula reduces to

$$
\left|\mathcal{B}^{\bullet}\right|=\frac{1}{3} 9^{n}+\frac{2}{3} 3^{n}-1+7|\mathcal{B}|
$$

In the following lemma we use a recursive relation to develop a formula for the size of $\left|\mathcal{B}^{\bullet}\right|$ when $v=3^{n}$.

Lemma 45. Let $m$ be a positive integer greater than or equal to 1 and define a sequence as follows:

$$
\begin{aligned}
b(n+1) & =\frac{1}{3} 9^{n}+\frac{2}{3} 3^{n}-1+7 b(n), \\
b(m) & =\beta .
\end{aligned}
$$

Then, for all $n \geq m$ :

$$
b(n)=\left[6.7^{n-m} \beta-9^{m} \cdot 7^{n-m}+3^{m} \cdot 7^{n-m}-7^{n-m}+9^{n}-3^{n}+1\right] / 6 .
$$

Proof. Substituting into $b(n+1)=\frac{1}{3} 9^{n}+\frac{2}{3} 3^{n}-1+7 b(n)$ we obtain

$$
\begin{aligned}
b(m+1) & =\frac{1}{3} 9^{m}+\frac{2}{3} 3^{m}-1+7 b(m) \\
& =\left[-7.9^{m}+7.3^{m}-7+6.7 \beta+9.9^{m}-3.3^{m}+1\right] / 6 \\
& =\left[6.7^{m+1-m} \beta-9^{m} \cdot 7^{m+1-m}+3^{m} \cdot 7^{m+1-m}-7^{m+1-m}+9^{m+1}-3^{m+1}+1\right] / 6
\end{aligned}
$$

as required. Thus the statement is true for $n=m+1$. Assume the statement is true for $n=k \geq m$ and we will prove it is true for $n=k+1$. That is, we will prove

$$
b(k+1)=\left[6.7^{k+1-m} \beta-9^{m} \cdot 7^{k+1-m}+3^{m} \cdot 7^{k+1-m}-7^{k+1-m}+9^{k+1}-3^{k+1}+1\right] / 6 .
$$

We know

$$
b(k+1)=\frac{1}{3} 9^{k}+\frac{2}{3} 3^{k}-1+7 b(k)
$$

and by the inductive hypothesis

$$
\begin{aligned}
b(k+1) & =\frac{1}{3} 9^{k}+\frac{2}{3} 3^{k}-1+7\left(\frac{1}{6}\left(6 \cdot 7^{k-m} \beta-9^{m} \cdot 7^{k-m}+3^{m} \cdot 7^{k-m}-7^{k-m}+9^{k}-3^{k}+1\right)\right) \\
& =\left[\left(6.7^{k+1-m} \beta-9^{m} \cdot 7^{k+1-m}+3^{m} \cdot 7^{k+1-m}-7^{k+1-m}+9^{k+1}-3^{k+1}+1\right)\right] / 6
\end{aligned}
$$

as required.

Corollary 46. If there exists a minimal defining set of order $3^{m}$, where $m \geq 2$, with volume $\beta$, then for all $n \geq m$ there exists a minimal defining set containing $b(n)$ triples where

$$
b(n)=\left[6.7^{n-m} \beta-9^{m} \cdot 7^{n-m}+3^{m} \cdot 7^{n-m}-7^{n-m}+9^{n}-3^{n}+1\right] / 6 .
$$

\subsection{Applications to Steiner triple Systems from affine geometries}

A Steiner triple system of order $3^{n}, n \geq 2$, denoted $S T S_{A}\left(3^{n}\right)$, from an affine geometry $A G(n, 3)$ can be obtained as follows. Let $V$ be a $n$-dimensional vector space, $n \geq 2$, over $G F(3)$, the Galois field of order 3. Define

$$
\mathcal{T}=\{\{x, y, z\} \mid x, y, z \in V, x \neq y \text { and } x+y+z=0\} .
$$

Then $(V, \mathcal{T})$ is a Steiner triple system of order $3^{d}$.

Gower in [7] and the present authors in [3] introduced minimal defining sets in $S T S_{A}\left(3^{n}\right)$ which correspond to collections of hyperplanes of the affine geometry $A G(n, 3)$. In 1997, Gower [7] obtained the following result. Here we give a different proof of this result. 
Corollary 47. There exists a minimal defining set in $S T S_{A}\left(3^{n}\right), n \geq 2$, having $b(n)$ triples where

$$
b(n)=\frac{1}{6}\left(3^{n}\left(3^{n}-1\right)-7^{n}+1\right) .
$$

Proof. When $n=2$ the Steiner triple system $(V, \mathcal{T})$ obtained from $A G(2,3)$ is the unique $S T S(9)$ given in Example 1. As Gower noted in [7] if we start the recursive construction explained in Construction 32 with this $S T S(9)$, then the resulting Steiner triple systems are isomorphic to $S T S_{A}\left(3^{n}\right)$ for some $n$. Now let $\mathcal{B}$ be as in Example 1. Then $\mathcal{B}$ and the partial Steiner latin square corresponding to $\mathcal{B}$ satisfy Conditions (1), (2) and (3) in Theorem 42. Now the result follows by Corollary 44 and Corollary 46 .

We make use of the following example in Corollary 49 below.

Example 48. (I) It is well-known that $\mathcal{B}=\{\{0,1,2\},\{0,3,6\},\{0,4,8\},\{3,4,5\},\{1,3,8\}\}$ is a minimal defining set in an $S T S_{A}\left(3^{2}\right)$ on element set $\{0,1,2, \ldots, 8\}$. Note that the element 7 does not occur in any triple of $\mathcal{B}$.

(II) A computer search shows that the following 65 triples form a minimal defining set in an $S T S_{A}\left(3^{3}\right)$ on element set $\{0,1,2 \ldots, 26\}$. Note that the element 26 does not occur in any triple.

\begin{tabular}{|c|c|c|c|c|}
\hline 2$\}$ & $6\}$ & $\{0$ & $\{0$, & $9,18\}$ \\
\hline 10,20$\}$ & $11,19\}$ & $\{0,12,24\}$ & $\{0,14,25\}$ & $\{0,15,21\}$ \\
\hline 3,8$\}$ & $4,7\}$ & $5,6\}$ & $9,20\}$ & $\{1,10,19\}$ \\
\hline 11,18$\}$ & $\{1,14,24\}$ & $\{1,16,22\}$ & $3,7\}$ & $4,6\}$ \\
\hline 5,8$\}$ & $9,19\}$ & $\{2,10,18\}$ & $\{2,11,20\}$ & $\{3$, \\
\hline 9,24$\}$ & $\{3,12,21\}$ & $\{3,13,23\}$ & $\{3,14,22\}$ & $\{3,15,18\}$ \\
\hline 16,20$\}$ & $\{4,10,25\}$ & $\{4,12,23\}$ & $\{4,13,22\}$ & $\{4,14,21\}$ \\
\hline 15,20$\}$ & $\{5,12,22\}$ & $\{5,13,21\}$ & $\{5,14,23\}$ & $\{6$, \\
\hline 9,21$\}$ & $\{6,12,18\}$ & $\{6,15,24\}$ & $9,22\}$ & $\{8,10,21\}$ \\
\hline 14,20 & $15,25\}$ & $\{8,16,24\}$ & $\{9,10,11\}$ & $\{9, \quad 12,15\}$ \\
\hline & $14,16\}$ & $12,17\}$ & $13,16\}$ & 14,1 \\
\hline 12 , & $\{11,13,15\}$ & $\{11,14,17\}$ & $\{12,13,14\}$ & 16 , \\
\hline 19 & $\{18,21,24\}$ & $\{20,21,25\}$ & $\{20,22,24\}$ & $\{21,22,23\}$ \\
\hline
\end{tabular}

The present authors in [3] obtained the following results. Similarly, one can also apply Corollary 44, Corollary 46 and Example 48 to prove these results.

Corollary 49. (I) There exists a minimal defining set in some $S T S_{A}\left(3^{n}\right), n \geq 2$, having $b(n)$ triples where

$$
b(n)=\frac{1}{6}\left(3^{n}\left(3^{n}-1\right)-7^{n-2}+1\right)-7^{n-1} .
$$

(II) There exists a minimal defining set in some $S T S_{A}\left(3^{n}\right), n \geq 3$, having $b(n)$ triples where

$$
b(n)=\frac{1}{6}\left(3^{n}\left(3^{n}-1\right)-7^{n-3}+1\right)-52.7^{n-3} .
$$

\subsection{Applications to Hall triple systems}

The Hall triple systems (HTS s) are the Steiner triple systems in which any three elements not all in the one triple generate an $S T S(9)$. It has been shown (see [1]) that the order of a $H T S$ is a power of three. An HTS which arises from an affine geometry is called abelian. Marshall Hall showed [10] that the smallest non-abelian example of HTS contains 81 elements. Moreover, there is (up to isomorphism) only one non-abelian HTS of order 81. Here we explain how one can construct such a HTS of order 81 . 
Let $V$ be a 4-dimensional space over the Galois field of order $3, G F(3)$. Let $e_{1}, e_{2}, e_{3}, e_{4}$ be a basis for $V$. For $x, y \in V$ we have $x=a_{1} e_{1}+a_{2} e_{2}+a_{3} e_{3}+a_{4} e_{4}$ and $y=b_{1} e_{1}+$ $b_{2} e_{2}+b_{3} e_{3}+b_{4} e_{4}$, where $a_{i}, b_{i} \in G F(3)$ for $i \in\{1,2,3,4\}$. Now define $x \circ y=z$, where $z=\left(a_{2}-b_{2}\right)\left(a_{3} b_{4}-a_{4} b_{3}\right) e_{1}-(x+y)$. It is now easy to show that either $x=y=z$ or $x, y$, and $z$ are pairwise distinct. Define

$$
\mathcal{T}=\{\{x, y, x \circ y\} \mid x, y \in V \text { and } x \neq y\}
$$

Then $(V, \mathcal{T})$ is the non-abelian $H T S$ of order 81.

The authors were able to find a minimal defining set with 680 triples for the non-abelian $H T S$ of order 81 (see the Appendix). Using computer programs we found that this minimal defining set and its corresponding partial Steiner latin square satisfy Conditions (1), (2) and (3) of Theorem 42. So this minimal defining set generates an infinite family of minimal defining sets of order $3^{n}, n \geq 4$, which uniquely determine a $S T S\left(3^{n}\right)$ not isomorphic to $S T S_{A}\left(3^{n}\right)$.

Corollary 50. In an $S T S\left(3^{n}\right)$ which is not isomorphic to $S T S_{A}\left(3^{n}\right), n \geq 4$, there exists a minimal defining set with $b(n)$ triples where

$$
b(n)=\left[3^{n}\left(3^{n}-1\right)-7^{n}+1\right] / 6 .
$$

\section{REFERENCES}

[1] R. H. Bruck, Contribution to the theory of loops, Transactions of the American Mathematical Society, 60 (1946) 245-354.

[2] C.J. Colbourn and A. Rosa, Triple Systems, Clarendon Press (Oxford) 1999.

[3] D. Donovan, A. Khodkar and A. P. Street, On minimal defining sets in AG(d,3), (submitted).

[4] B. Ganter and H. Werner, Co-ordinatizing Steiner systems, Topics on Steiner systems (C.C. Lindner and A. Rosa, eds), Annals of Discrete Mathematics 7, 1980, pp. 3-24.

[5] R.A.H. Gower, Minimal defining sets in a family of Steiner triple systems, Australasian Journal of Combinatorics 8 (1993), 55-73.

[6] R.A.H. Gower, Critical sets in products of latin squares, Ars Combinatoria 55 (2000), 293-317.

[7] R.A.H. Gower, Defining sets for the Steiner triple systems from affine spaces, Journal of Combinatorial Designs 5 (1997), 155-175.

[8] K. Gray, On the minimum number of blocks defining a design, Bulletin of the Australian Mathematical Society 41 (1990), 97-112.

[9] B.D. Gray and C. Ramsay, Some results on defining sets of t-designs, Bulletin of the Australian Mathematical Society 59 (1999), 203-215.

[10] M. Hall, Automorphisms of Steiner triple systems, IBM Journal of Research and Development (1960), $460-472$.

[11] A.D. Keedwell, Critical sets for latin squares, graphs and block designs: a survey, Congressus Numerantium 113 (1996), 231-245.

[12] G.B. Khosrovshahi and H.R. Maimani, On 2- $v, 3)$ Steiner trades of small volumes, Ars Combinatoria 52 (1999), 199-220.

\section{Appendix}

This is a minimal defining set in the non-abelian HTS of order 81 , having 680 triples. 


\begin{tabular}{|c|c|c|c|c|c|c|}
\hline & $6\}$ & & & & & \\
\hline 12,24$\}$ & $, 13,26\}$ & $25\}$ & $\{15,21\}$ & $\{0,16,23\}$ & $\{0,17,22\}$ & \\
\hline & & & & & & \\
\hline & & & & & & \\
\hline 64$\}$ & & & & & $0,19\}$ & \\
\hline & & & & & & \\
\hline & & & & & & \\
\hline & & & & & & \\
\hline & & & & & & \\
\hline & & & & & & \\
\hline & & & & & & \\
\hline & & & & & & \\
\hline & & & & & & \\
\hline & & & & & & \\
\hline & & & & & & \\
\hline & & & & & & \\
\hline & & & & & & \\
\hline & & & & & & \\
\hline & & & & & & \\
\hline & & & & & & \\
\hline & & & & & & \\
\hline & & & & & & \\
\hline & & & & & & \\
\hline & & & & & & \\
\hline & & & & & & \\
\hline & & & & & & \\
\hline & & & & & & \\
\hline & & & & & & \\
\hline & & & & & & \\
\hline & & & & & & \\
\hline & & & & & & \\
\hline & & & & & & \\
\hline & & & & & & \\
\hline & & & & & & \\
\hline & & & & & & $73\}$ \\
\hline & & & & & & \\
\hline & & & & & & \\
\hline & & & & & & \\
\hline & & & & & & \\
\hline & & & & & & \\
\hline & & & & & & \\
\hline & & & & & & \\
\hline & & & & & & $59\}$ \\
\hline & & & & & & \\
\hline & & & & & & \\
\hline & $\{12$, & & $27,78\}$ & $30,77\}$ & $31,76\}$ & $2,75\}$ \\
\hline & & & 10 & & $\{13$, & 18 \\
\hline & & & & & & \\
\hline & & & & & & 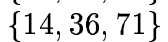 \\
\hline
\end{tabular}


$\{14,37,70\}\{14,38,69\}\{14,39,67\}\{14,40,66\}\{14,41,68\}\{14,42,63\}\{14,43,65\}$ $\{14,44,64\}\{14,47,60\}\{14,48,58\}\{14,49,57\}\{14,50,59\}\{15,16,17\}\{15,31,72\}$ $\{15,33,78\}\{15,36,68\}\{15,37,67\}\{15,38,66\}\{15,39,64\}\{15,40,63\}\{15,41,65\}$ $\{15,42,69\}\{15,43,71\}\{15,44,70\}\{15,46,58\}\{15,50,56\}\{15,51,60\}\{16,28,75\}$ $\{16,32,73\}\{16,34,79\}\{16,36,67\}\{16,37,66\}\{16,38,68\}\{16,39,63\}\{16,40,65\}$ $\{16,41,64\}\{16,42,71\}\{16,43,70\}\{16,44,69\}\{16,48,54\}\{16,52,61\}\{17,29,76\}$ $\{17,36,66\}\{17,37,68\}\{17,38,67\}\{17,39,65\}\{17,40,64\}\{17,41,63\}\{17,42,70\}$ $\{17,43,69\}\{17,44,71\}\{17,45,57\}\{18,19,20\}\{18,21,24\}\{18,22,26\}\{18,23,25\}$ $\{18,27,63\}\{18,28,65\}\{18,29,64\}\{18,36,54\}\{18,37,56\}\{18,38,55\}\{18,45,72\}$ $\{18,46,74\}\{18,47,73\}\{19,21,26\}\{19,22,25\}\{19,23,24\}\{19,27,65\}\{19,28,64\}$ $\{19,29,63\}\{19,31,71\}\{19,33,67\}\{19,36,56\}\{19,37,55\}\{19,38,54\}\{19,39,60\}$ $\{19,43,57\}\{19,45,74\}\{19,46,73\}\{19,47,72\}\{19,48,78\}\{19,53,77\}\{20,21,25\}$ $\{20,22,24\}\{20,23,26\}\{20,27,64\}\{20,28,63\}\{20,29,65\}\{20,32,69\}\{20,34,68\}$ $\{20,36,55\}\{20,37,54\}\{20,38,56\}\{20,40,61\}\{20,44,58\}\{20,45,73\}\{20,46,72\}$ $\{20,47,74\}\{20,49,79\}\{20,51,75\}\{21,22,23\}\{21,30,66\}\{21,31,68\}\{21,32,67\}$ $\{21,34,63\}\{21,39,57\}\{21,40,59\}\{21,41,58\}\{21,43,54\}\{21,48,75\}\{21,49,77\}$ $\{21,50,76\}\{22,29,71\}\{22,30,68\}\{22,31,67\}\{22,32,66\}\{22,37,60\}\{22,39,59\}$ $\{22,40,58\}\{22,41,57\}\{22,44,55\}\{22,46,78\}\{22,48,77\}\{22,49,76\}\{22,50,75\}$ $\{22,51,72\}\{23,27,69\}\{23,30,67\}\{23,31,66\}\{23,32,68\}\{23,33,65\}\{23,38,61\}$ $\{23,39,58\}\{23,40,57\}\{23,41,59\}\{23,47,79\}\{23,48,76\}\{23,49,75\}\{23,50,77\}$ $\{23,52,73\}\{24,25,26\}\{24,27,67\}\{24,30,65\}\{24,33,69\}\{24,36,58\}\{24,39,56\}$ $\{24,42,60\}\{24,45,76\}\{24,48,74\}\{24,51,78\}\{25,28,68\}\{25,31,63\}\{25,34,70\}$ $\{25,37,59\}\{25,40,54\}\{25,43,61\}\{25,46,77\}\{25,49,72\}\{25,52,79\}\{27,28,29\}$ $\{27,30,33\}\{27,31,35\}\{27,32,34\}\{27,36,45\}\{27,37,47\}\{27,38,46\}\{27,39,52\}$ $\{27,40,51\}\{27,41,53\}\{27,42,50\}\{27,43,49\}\{27,44,48\}\{28,30,35\}\{28,31,34\}$ $\{28,32,33\}\{28,36,47\}\{28,37,46\}\{28,38,45\}\{28,39,51\}\{28,40,53\}\{28,41,52\}$ $\{28,42,49\}\{28,43,48\}\{28,44,50\}\{29,30,34\}\{29,31,33\}\{29,32,35\}\{29,36,46\}$ $\{29,37,45\}\{29,38,47\}\{29,39,53\}\{29,40,52\}\{29,41,51\}\{29,42,48\}\{29,43,50\}$ $\{29,44,49\}\{30,31,32\}\{30,36,53\}\{30,37,52\}\{30,38,51\}\{30,39,48\}\{30,40,50\}$ $\{30,41,49\}\{30,42,46\}\{30,43,45\}\{30,44,47\}\{31,36,52\}\{31,37,51\}\{31,38,53\}$ $\{31,39,50\}\{31,40,49\}\{31,41,48\}\{31,42,45\}\{31,43,47\}\{31,44,46\}\{32,36,51\}$ $\{32,37,53\}\{32,38,52\}\{32,39,49\}\{32,40,48\}\{32,41,50\}\{32,42,47\}\{32,43,46\}$ $\{32,44,45\}\{33,34,35\}\{33,36,49\}\{33,37,48\}\{33,38,50\}\{33,39,47\}\{33,40,46\}$ $\{33,41,45\}\{33,42,51\}\{33,43,53\}\{33,44,52\}\{34,36,48\}\{34,37,50\}\{334,38,49\}$ $\{34,39,46\}\{34,40,45\}\{34,41,47\}\{34,42,53\}\{34,43,52\}\{34,44,51\}\{35,36,50\}$ $\{35,37,49\}\{35,38,48\}\{35,39,45\}\{35,40,47\}\{35,41,46\}\{35,42,52\}\{35,43,51\}$ $\{35,44,53\}\{36,37,38\}\{36,39,42\}\{36,40,44\}\{36,41,43\}\{37,39,44\}\{37,40,43\}$ $\{37,41,42\}\{38,39,43\}\{38,40,42\}\{38,41,44\}\{39,40,41\}\{42,43,44\}\{45,46,47\}$ $\{45,48,51\}\{45,49,53\}\{45,50,52\}\{46,48,53\}\{46,49,52\}\{46,50,51\}\{47,48,52\}$ $\{47,49,51\}\{47,50,53\}\{48,49,50\}\{51,52,53\}\{54,55,56\}\{54,57,60\}\{54,58,62\}$ $\{54,59,61\}\{54,63,72\}\{54,64,74\}\{54,65,73\}\{54,71,77\}\{55,57,62\}\{55,58,61\}$ $\{55,59,60\}\{55,63,74\}\{55,64,73\}\{55,65,72\}\{55,67,78\}\{55,69,75\}\{56,57,61\}$ $\{56,58,60\}\{56,59,62\}\{56,63,73\}\{56,64,72\}\{56,65,74\}\{56,68,79\}\{57,58,59\}$ $\{57,63,79\}\{57,66,75\}\{57,67,77\}\{57,68,76\}\{57,71,72\}\{58,66,77\}\{58,67,76\}$ $\{58,68,75\}\{58,69,73\}\{59,65,78\}\{59,66,76\}\{59,67,75\}\{59,68,77\}\{60,61,62\}$ $\{60,63,77\}\{60,67,72\}\{60,69,78\}\{61,64,75\}\{61,68,73\}\{61,70,79\}\{63,64,65\}$ $\{63,66,69\}\{63,67,71\}\{63,68,70\}\{64,66,71\}\{64,67,70\}\{64,68,69\}\{65,66,70\}$ $\{65,67,69\}\{65,68,71\}\{66,67,68\}\{69,70,71\}\{72,73,74\}\{72,75,78\}\{173,76,79\}$ $\{75,76,77\}$ 
Lemma 51. Let $(V, \mathcal{B})$ be a $P S T S(v)$ contained in the $S T S(v)(V, \mathcal{T})$. Moreover, let $P$ be the partial sloop corresponding to $(V, \mathcal{B})$ and let $L$ be the sloop corresponding to $(V, \mathcal{T})$. Then $P^{*}$ is the partial sloop corresponding to $\left(V^{*}, \mathcal{B}^{*}\right)$ and $L^{*}$ is the sloop corresponding to $\left(V^{*}, \mathcal{T}^{*}\right)$.

Proof. Let $P^{\prime}$ be the partial sloop associated with $\left(V^{*}, \mathcal{B}^{*}\right)$ and let $L^{\prime}$ be the sloop associated with $\left(V^{*}, \mathcal{T}^{*}\right)$ (see Definition 7 ). We need to show that $P^{\prime}=P^{*}$ and $L^{\prime}=L^{*}$. We only prove $P^{\prime}=P^{*}$. The other equality can be proved in a similar manner. Let $i, j, k \in V^{*}$ and $P^{\prime}(i, j)=k$. Since $P^{*}$ and $P^{\prime}$ are both symmetric we only prove $P^{*}(i, j)=P^{\prime}(i, j)$ for $i \leq j$.

Case 1: $i=j$. Then $P^{\prime}(i, i)=2 v+1$ and $i$ occurs in a triple of $\mathcal{B}^{*}$ by Definition 7. If $i \in V$ then $i$ occurs in a triple of $\mathcal{B}$ by Construction 10 . So $P(i, i)=v$ by Definition 7 and $\hat{P}(i, i)=v$ by Construction 13. Therefore $P^{*}(i, i)=2 v+1$ by Construction 13. If $i=v$ then $P(i, i)=v$ by Definition 7. So $\hat{P}(i, i)=v$ and $P^{*}(i, i)=2 v+1$ by Construction 13 . If $v+1 \leq i \leq 2 v+1$ then $i=i^{\prime}+v+1$ where $i^{\prime} \in V$ and $i^{\prime}$ occurs in a triple of $\mathcal{B}$. So $P\left(i^{\prime}, i^{\prime}\right)=v$ and $\hat{P}(i, i)=v$. Therefore $P^{*}(i, i)=2 v+1$ by Construction 13 .

Case 2: $i<j$. Let $P^{\prime}(i, j)=k$. If $i, j \in V$ then $\{i, j, k\} \in \mathcal{B}^{*}$ by Definition 7 and $\{i, j, k\} \in \mathcal{B}$ by Construction 10 . So we have $P(i, j)=k$. Now Construction 13 shows that $P^{*}(i, j)=\hat{P}(i, j)=P(i, j)=k$ as required.

If $i \in V$ and $j=v$ then $i$ occurs in a triple of $\mathcal{B}$ and $k=i+v+1$ by Construction 10 . So $P^{*}(i, j)=P^{*}(i, v)=\hat{P}(i, 2 v+1)=i+v+1=k$ by Construction 13 as required.

If $i \in V$ and $v+1 \leq j<2 v+1$ then either $\left\{i, j^{\prime}, k^{\prime}\right\} \in \mathcal{B}, j=j^{\prime}+v+1$ and $k=k^{\prime}+v+1$, or $\{i, v, i+v+1\} \in \mathcal{B}^{*}, j=i+v+1, k=v$ and $i$ occurs in a triple of $\mathcal{B}$. In the first case $P\left(i, j^{\prime}\right)=k^{\prime}$ which leads to $\hat{P}(i, j)=k$. So $P^{*}(i, j)=k$. In the second case we have $P(i, i)=v$ and $\hat{P}(i, i+v+1)=v$. So $P^{*}(i, j)=P^{*}(i, i+v+1)=\hat{P}(i, i+v+1)=v=k$ as required.

If $i \in V$ and $j=2 v+1$ then $P^{\prime}(i, j)=i$ and $i$ occurs in a triple of $\mathcal{B}^{*}$. So $i$ also occurs in a triple of $\mathcal{B}$. This leads to $P^{*}(i, j)=P^{*}(i, 2 v+1)=\hat{P}(i, v)=P(i, v)=i$ as required.

If $i=v$ and $v+1 \leq j<2 v+1$ then $\left\{j^{\prime}, v, j^{\prime}+v+1\right\} \in \mathcal{B}^{*}$ where $j=j^{\prime}+v+1$ and $j^{\prime}$ occurs in a triple of $\mathcal{B}$. (Note that in this case $k=j^{\prime}$.) So $P\left(v, j^{\prime}\right)=j^{\prime}$ by Definition 7 and $\hat{P}\left(2 v+1, j^{\prime}+v+1\right)=j^{\prime}$ by Construction 13 . So $P^{*}(i, j)=P^{*}(v, j)=j^{\prime}$ as required.

If $i=v$ and $j=2 v+1$ then $P^{\prime}(i, j)=i$ by Definition 7 . Since $i=v$ we have $P(v, v)=v$ and $\hat{P}(2 v+1,2 v+1)=v=i$. So $P^{*}(i, j)=P^{*}(v, 2 v+1)=v=i$.

Finally let $v+1 \leq i<j<2 v+1$. Then $i=i^{\prime}+v+1, j=j^{\prime}+v+1$ and $\left\{i^{\prime}, j^{\prime}, k\right\} \in \mathcal{B}$ by Construction 10. Now applying Construction 13 we see that $P^{*}(i, j)=\hat{P}(i, j)=P\left(i^{\prime}, j^{\prime}\right)=$ $k$ as required. 